\title{
Espacio y movimiento del cuidador de la persona con discapacidad motora
}

\author{
Sandra Hernández Corral,* Ma. Guadalupe Ojeda Vargas**
}

\begin{abstract}
RESUMEN
Objetivo: Analizar y describir el espacio y movimiento del cuidador de la persona con discapacidad motora. Metodología: Investigación de abordaje cualitativo y de tipo descriptiva exploratoria; los informantes fueron cuatro cuidadores de personas con discapacidad motora hospitalizados en el Servicio de Rehabilitación Neurológica; los datos fueron recolectados mediante entrevista a profundidad, la cual fue grabada, transcrita y analizada sobre la base de análisis de discurso. Resultados: Emergieron dos categorías teóricas analíticas: espacio y movimiento, con las subcategorías para espacio: necesidad de bañar a la persona y necesidad de hacerle sus ejercicios a la persona, y la segunda categoría movimiento con la subcategoría, movimiento ascendente. Conclusiones: El espacio del cuidador de la persona con discapacidad motora se centra en las actividades de cuidado, como son: baño, alimentación, comunicación, movilización y terapias, y el movimiento se manifiesta a través del proceso interactivo de cuidar.
\end{abstract}

Palabras clave: Cuidadores, discapacidad, tiempo, espacio, movimiento.

\section{Space and movement of the caregiver of the person with motor disabilities}

\begin{abstract}
Objective: To analyze and describe the motion space and caregivers of people with motor disabilities. Methodology: Qualitative research approach was exploratory and descriptive; the informants were four caregivers of people with motor disabilities hospitalized in the Neurological Rehabilitation Service, data were collected through in-depth interview, which was recorded, transcribed and analyzed on the basis discourse analysis. Results: Analytical theory emerged two categories: space and movement, with the subcategories to space: the need to bathe the person and need to give the person exercises and the second category with the subcategory movement, upward movement. Conclusions: The space of the caregiver of the person with motor disabilities focuses on self-care activities such as: bathing, feeding, communication, mobilization and therapies, and movement manifests itself through the interactive process of care.
\end{abstract}

Key words: Caregivers, disability, time, space, movement.

\footnotetext{
* M.E. Subjefa de Educación e Investigación en Enfermería del Instituto Nacional de Rehabilitación. Alumna del Programa de Doctorado en Ciencias de la Enfermería, Convenio Universidad Nacional de Trujillo-Universidad Nacional Autónoma de México-México.

Parte de la Tesis de Doctorado en Ciencias de Enfermería "Tiempo, espacio y movimiento de los cuidadores de personas con discapacidad". Universidad Nacional de Trujillo-Perú-Universidad Nacional Autónoma de México-México.

**Doctora en Enfermería Psiquiátrica, profesora invitada del Convenio UNAM-UNT y profesora de tiempo completo, Departamento de Enfermería y Obstetricia, División de Ciencias de la Salud e Ingenierías, Campus Celaya Salvatierra, Universidad de Guanajuato.

Correspondencia: Sandra Hernández Corral. Calzada México-Xochimilco Núm. 289, Col. Arenal de Guadalupe. 14389, Del. Tlalpan.

Tel: 5999 1000, ext. 12210, E-mail: shcorral@gmail.com
}

Este artículo puede ser consultado en versión completa en http://www.medigraphic.com/enfermerianeurologica 


\section{INTRODUCCIÓN}

$\mathrm{S}$ e estima que más de mil millones de personas viven con algún tipo de discapacidad; según las estimaciones de la población mundial en el 2010, alrededor del $15 \%$ de la población mundial. ${ }^{1}$ En América Latina y el Caribe, en el 2009 habían por lo menos 50 millones de personas con algún tipo de discapacidad, según cifras reportadas por el Banco Mundial. ${ }^{2}$

En México existen 5’739,270 personas con alguna discapacidad física o mental para realizar actividades de la vida cotidiana. El $40.7 \%$ de las personas con alguna discapacidad tienen entre 60 y 84 años de edad, el 32.8\% se encuentra en la edad adulta entre 30 y 59 años y el porcentaje menor está representado por la población de 85 años y más. Cabe mencionar que dos de cada diez individuos con discapacidad tiene menos de 30 años. ${ }^{3}$

La discapacidad que presenta con mayor frecuencia la población mexicana es la que se relaciona con la movilidad, ya que alrededor de la mitad de las limitaciones declaradas se refieren a caminar o moverse (58.3\%). ${ }^{3}$ La Encuesta Nacional de Salud y Nutrición $2012^{4}$ confirma que la discapacidad motora es la más frecuente y está presente en 2'442,400 hombres y 3'036,100 mujeres; estas personas manifestaron tener dificultad para caminar, moverse, subir o bajar escaleras.

En situaciones como éstas, la mayoría de los familiares (cuidadores) proporciona el apoyo; sin embargo, se les demanda compromiso y dedicación, ya que deberá invertir tiempo para proporcionar cuidado encaminado a la satisfacción de las necesidades básicas.

Se han realizado diversas investigaciones que abordan el tiempo y espacio dedicados al cuidado de las personas con discapacidad; el $91 \%$ de los cuidadores de niños refieren cuidarlos desde el momento del diagnóstico; respecto al tiempo de experiencia como cuidador, el $78 \%$ lleva 37 meses y el $61 \%$ dedica las 24 horas del día al cuidado. ${ }^{5}$ Las madres manifestaron un carácter sobrenatural del cuidado, declarando que la cantidad de tiempo y la intensidad de dedicación para los niños exigen mucho más que la práctica natural de cuidarlos. ${ }^{6}$

En los adultos mayores dependientes el cuidador dedica más tiempo y lo hace de manera continua durante el día.? En Chile, en el 2012, Flores, Rivas y Seguel ${ }^{8}$ observaron que el $46.3 \%$ de los cuidadores dedicó de 21 a 24 horas al cuidado y $53 \%$ lleva más de cinco años realizando esta tarea, mientras que Espinoza y Jofré ${ }^{2}$ mencionan que los cuidadores de pacientes dependientes inscritos en el programa de atención domiciliaria han realizado esta labor desde hace nueve años en promedio, dedicando 17 horas del día a esta tarea. Por otra parte, el tiempo dedicado al cuidado de adultos mayores en el domicilio es de más de tres años en el $46.6 \% .^{10}$ En adultos mayores frágiles, los cuidadores invierten como mínimo tres horas diarias. Además, precisan dividir ese cuidado con los quehaceres domésticos, el trabajo y el cuidado de los hijos, entre otras actividades. ${ }^{11}$ Con relación al movimiento, Schnaider, Silva y Pereira ${ }^{12}$ mencionan que una de las actitudes del cuidador en el arte de cuidar es acompañar los cambios y movimientos de vida de sí mismo y de los demás. La observación día con día, característica de la relación paciente-cuidador se revela: en cuanto los cuidadores creen que los pacientes son cada vez más dependientes para las actividades de la vida diaria, no consiguen percibir lo que pueden hacer solos; ésta es una manifestación de sobreprotección; por otra parte, los pacientes pueden sentirse una carga para los cuidadores. ${ }^{12}$ La construcción de significados por los seres humanos es un proceso interpretativo que da lugar a la acción; cuando se enfrenta al mundo se acepta hacerle frente a todo tipo de situaciones. Para llegar a tal intento, el humano reflexiona, interpreta y determina su acción a la luz de sus reflexiones. Esto significa que ser cuidador de un familiar dependiente significa asumir tareas que antes no conocía y que afectan directamente su cotidianidad. Inconscientemente, analiza y obtiene respuestas a ella. Todo lo que aprende está cambiando, ocurren cambios en su vida rutinaria y tiene que lidiar con esto todos los días. ${ }^{13}$

El referencial teórico del espacio y movimiento del cuidador de la persona con discapacidad motora se basó en Henri Bergson y el cuidado en Waldow, autores que dieron fundamento teórico a los resultados encontrados.

De acuerdo con Bergson, el carácter mismo de los fenómenos psíquicos está en lo cualitativo (y por tanto no pueden ser mensurados cuantitativamente) y cada intuición (cualidad) es irrepetible, irreversible y no puede ordenarse en una instancia reversible y homogénea en la que prima la yuxtaposición, pues se interpretan y se funden entre sí formando un fluir único, una continuidad inseparable (duración). Aquí está la distinción entre tiempo espacializado, que es el tiempo físico que contempla la ciencia, y el tiempo auténtico, la duración de la vida interior de la conciencia, el puro movimiento en el que no pueden ser diferenciados los momentos como estados distintos. ${ }^{14}$

El tiempo real, "o tiempo vivido" o lo que podría ser el tiempo de Bergson, no es el espacio del tiempo, este vacío en el que los acontecimientos se sucederían. El filósofo propone desviemos la mirada y consideremos los propios acontecimientos sean físicos o psíquicos. Es aquí donde se descubre el tiempo real, cuyas propiedades fundamentales son sucesión, continuidad y duración. ${ }^{15}$

Bergson ${ }^{15}$ dice que el movimiento se compone de puntos, pero que comprende, además, el pasaje obscuro, misterioso, 
de una posición a la posición siguiente. Las posiciones del móvil, no son partes del movimiento, son puntos del espacio que, según el supuesto, subtiende al movimiento. Este espacio inmóvil y vacío, sólo concebido, nunca percibido, tiene precisamente todo el valor de un símbolo.

Bergson, entre sus proposiciones relacionadas con el movimiento, menciona que hay una realidad exterior y no obstante dada inmediatamente a nuestro espíritu, por lo que considera que esta realidad es movilidad. No existen cosas hechas, sino sólo cosas que se hacen; no estados que se mantienen, sino estados que cambian. La conciencia que tenemos de nuestra propia persona, en su continuo fluir, nos introduce en el interior de una realidad sobre el modelo de la cual debemos representarnos las otras. Toda realidad es tendencia a un cambio de dirección en estado naciente. ${ }^{15}$ Un movimiento repetitivo, descendente y un movimiento ascendente que es creación, "elaboración continua de lo absolutamente nuevo". Ambas tendencias se encuentran en la evolución de la vida. En esta evolución se verifica, en efecto, un movimiento ascendente denominado impulso creador que produce formas cada vez más complejas, cada vez más perfectas, junto a un movimiento descendente de retroceso. La evolución no siempre avanza, a veces retrocede. ${ }^{16}$

Para entender los procesos de cuidado, Waldow, en el 2008, ${ }^{17}$ menciona que el cuidado es una forma de ser hombre, y tiene un significado a partir del propio hombre, incluyendo comportamientos, actitudes, valores y principios que son vividos por las personas en determinadas circunstancias; sin embargo, se refiere al ser. El ser humano nace con un potencial de cuidado, lo que significa que todas las personas son capaces de cuidar. Evidentemente, esta capacidad podrá desarrollarse conforme las circunstancias en que sea ejecutada durante las diversas etapas de la vida.

Por otra parte, el cuidar es un compromiso, significa ayudar a otros a crecer en su integridad y unidad, manteniendo su dignidad y su singularidad. Por lo tanto, el cuidar es un proceso interactivo que sólo se establece en la relación con el otro; también se considera transformador porque ambos crecen. El modo de ser del cuidado involucra una relación no sólo de sujeto-objeto, sino de sujeto-sujeto. ${ }^{17}$

La discapacidad es un fenómeno que va en aumento en la población a nivel mundial y específicamente en México; esto genera la necesidad de contar con cuidadores que apoyen la rehabilitación de este tipo de personas; se ha comprobado que una adaptación inefectiva del cuidador lo lleva a un estado de sobrecarga poniendo en riesgo su salud.

Ante este escenario, los cuidadores utilizan sus recursos para vivir la nueva situación de cuidado cambiante; de ahí la importancia que tiene para el profesional de enfermería analizar el espacio y el movimiento en el que ocurre su nuevo rol, con la finalidad de establecer programas de cuidado encaminados a promover un cambio desde sus propias vivencias, de tal forma que le permita desempeñar su rol de manera eficiente y al mismo tiempo facilitar la transición a la vida comunitaria, mediante acciones que promuevan la autonomía y permitan que las personas con discapacidad y sus familiares participen en actividades económicas y sociales.

Desde esta perspectiva, se planteó la pregunta de investigación: ¿Cómo es el espacio y movimiento de los cuidadores de la persona con discapacidad motora?

Los objetivos planteados fueron: describir y analizar el espacio y el movimiento de los cuidadores de personas con discapacidad motora.

\section{METODOLOGÍA}

Es una investigación de abordaje cualitativo de tipo descriptivo-exploratorio desarrollada en el Servicio de Rehabilitación Neurológica de un Hospital de Tercer Nivel. Participaron, hasta el momento, cuatro cuidadores de personas con discapacidad motora. Los criterios de elegibilidad fueron: ser cuidador de la persona con discapacidad motora, de ambos géneros, con edad mayor a 18 años, que proporcionaran cuidado a la persona hospitalizada.

Para la realización de las entrevistas en el hospital, se dio a conocer a los cuidadores el objetivo de la investigación y su relevancia científica y social; fueron también orientados en cuanto a la entrevista, la libertad de participar o no en el estudio, la protección de la identidad a través de un pseudónimo y el secreto de los datos obtenidos; así mismo se esclarecieron las dudas. Posterior a su autorización, los participantes firmaron el consentimiento informado.

Previo a realizar la entrevista, se acordó el horario y el lugar adecuados; se estableció una relación de respeto y empatía. La recolección de la información se realizó de febrero a mayo del 2013 por medio de entrevista a profundidad, mediante una pregunta semiestructurada diseñada con el tópico del objeto de estudio que es el tiempo, espacio y movimiento. Se caracterizó al participante y se formuló la pregunta: ¿Cómo han transcurrido desde el inicio hasta el día de hoy los cuidados que viene ofreciendo a su familiar? También se realizaron notas de campo para registrar el lenguaje no verbal.

Las respuestas fueron audiograbadas, lo que permitió apreciar los testimonios del participante. El procesamiento de datos fue por medio del análisis del discurso. Las narraciones de los cuidadores participantes fueron analizadas de acuerdo con el abordaje teórico. Las entrevistas fueron transcritas literalmente tal como fueron expresadas por los sujetos (texto EMIC); luego se realizó la reducción de los datos a texto ETIC sin perder la esencia del mensaje. En seguida se organizaron, clasificaron, redujeron a unidades 
mínimas y se clasificaron los testimonios semejantes y relevantes para identificar las categorías y subcategorías que emergieron del análisis. Para el rigor científico se consideraron los criterios: credibilidad, confiabilidad, confirmabilidad y transferibilidad.

La investigación se desarrolló aplicando los principios científicos y éticos conforme a lo establecido en la Ley General de Salud y su reglamento en materia de investigación, respetando los principios de confidencialidad, anonimato y privacidad. Se obtuvo el consentimiento de los participantes para la transcripción, análisis y publicación de los discursos y se respetó la confidencialidad de la información obtenida.

\section{RESULTADOS Y DISCUSIÓN}

Participaron en el estudio cuatro cuidadores, de los cuales tres fueron mujeres y un hombre, entre 38 y 58 años de edad, todos casados. El nivel de escolaridad fue uno con primaria, dos con secundaria y uno con preparatoria. Dos desempeñan actividades laborales externas al ambiente doméstico y dos se dedican a las actividades del hogar. De los cuatro, dos eran hijos, una esposa y una hermana. El tipo de discapacidad motora de las personas que cuidan fueron dos con síndrome de Guillen Barré, uno con lesión medular completa con nivel neurológico T10 y uno con lesión medular incompleta con nivel neurológico T1.

Posterior al análisis e interpretación de los cuatro discursos, se derivaron dos categorías teóricas analíticas: espacio y movimiento, la primera con las subcategorías: necesidad de bañar a la persona y necesidad de hacerle sus ejercicios a la persona. En la segunda categoría: movimiento, con la subcategoría: movimiento ascendente.

\section{Espacio}

El espacio es considerado como el tiempo y el movimiento que realizan los cuidadores a partir de que su familiar se convierte en paciente con discapacidad motora. En este espacio, la necesidad de baño es fundamental. El de esponja, en la camilla o en la silla son las formas que los cuidadores describen para esta actividad, la cual es aprendida por observación o mediante enseñanza de una enfermera; con el tiempo, los cuidadores van ajustando la forma de realizar esta actividad y con la ayuda que la persona con discapacidad les pueda proporcionar.

\section{Necesidad de bañar a la persona}

[...] "Allá se le daba baño de esponja, me ayudaban las enfermeras o enfermeros o más bien ellos se lo daban y yo nomás observaba." [...] "Aquí sí me enseñan cómo bañarlo en la camilla pero una sola vez y entonces no se me graba muy bien, pero lo baño." [...] (Lalo)

[...] "Cuando llegué al hospital era en la camilla, no podía sentarse para nada y sí era más difícil tanto para él como para mí, pero ahora ya es mucho mejor sentado y él ya se puede tallar, solamente le pongo agua y él se baña, lo que ya no alcanza a lavarse yo le lavo.” [...](Lola)

[...] "En neurología me enseñaron las enfermeras cómo bañarlo, los camilleros lo ponían en la silla y lo metían a bañar en la regadera, le tallaba yo la cabeza, su cara, sus dientes, le bañaba yo todo el cuerpo, lo enjuagaba; ya que acababa lo secaba, como un bebé; para mí fue como un bebé." [...] "Ahora es diferente porque él ya se lava los dientes, se enjuaga la cara, se seca o hace el propósito de secar su cabeza, antes no lo hacía."[...](Lili)

[...] "Al principio según se bañaba pero en la cama, no la bañaba, más bien la limpiaba y ya nada más disque se mojaba la cabeza, ¡no!, ahora en la camilla de baño la baño, iy sí se baña toda completa!, jun baño normal! y ella me ayuda, cuando se tiene que voltear ella mueve la cadera o la pierna y ella ya me ayuda con los movimientos para bañarla.” [...] (Lupe)

\section{Necesidad de hacerle ejercicios a la persona}

Los cuidadores manifestaron cómo surge la necesidad de hacerle ejercicios a la persona con discapacidad en diferentes espacios.

[...] "En neurología, como no se podía mover, me enseñó la doctora cómo hacerle sus terapias (moverle las manos, subírselas, bajárselas, en círculo, los pies estirárselos, sobárselos, doblárselos para arriba para abajo." [...] "En la casa ya le hacía más ejercicios, con más confianza; en el hospital me daba pena agarrarle aqui en las piernas (se toca la entrepierna) o sobárselas y allá en la casa ya se las hacía." [...] "Ahora ya los hace él mismo. Ya se voltea de la cama de un movimiento a otro, ya se jala de la silla, mueve más los pies y está haciendo el propósito de subir los pies, de apoyarlos en la silla de ruedas. Ahora ya hace más cosas que antes." [...] (Lili)

Los seres humanos fijan involuntariamente en un punto en el espacio cada uno de los momentos con los que cuentan, y es solamente en esta condición cuando las unidades abstractas forman una suma. Sin duda, es posible concebir los momentos sucesivos del tiempo con independencia del espacio; pero cuando se añade al instante actual los que le 
preceden, como ocurre cuando se adicionan unidades, no es con estos instantes mismos con los que se opera, puesto que se desvanecen para siempre, sino con la huella duradera que nos parecen haber dejado en el espacio al atravesarlo. ${ }^{18}$

\section{El movimiento}

\section{En el movimiento ascendente surge la paciencia}

El cuidar implica un movimiento en dirección de algo o alguien que es motivo de interés o preocupación. El ejercicio de cuidar es una acción que posee una direccionalidad concreta y determinada; nos mueve y nos impulsa a hacer algo; se trata de una acción moral objetiva, para aliviar, satisfacer, ayudar, confortar y apoyar. Abarca, además de procedimientos y actividades técnicas, acciones y comportamientos que favorecen; no sólo es estar con, sino también el ser con. Señaladas por los cuidadores con las características de, se requiere de mucha calma y/o paciencia, tal como lo expresan:

[...] "Tener calma, más que nada calma y paciencia hacia el paciente; porque él está enfermo; nosotros estamos bien. Es tener mucha calma y ayudarlo en todo, todo lo que se pueda y esté de nuestra parte. Es algo muy fundamental tener calma, no perderla, ayudarle más en lo que se puede." (Lalo)

[...] "Que le tengan mucha paciencia a sus pacientes y que lo hagan con mucho cariño y amor a sus pacientes, porque si uno se desespera en vez de ayudarlos los perjudicamos." [...] (Lili)

[...] "La verdad, yo pensé que no iba a tener paciencia, porque para eso se necesita mucha paciencia, (...) yo siempre he sido muy mandona, quiero que las cosas las hagan rápido y a mi modo y aquí hay que tener paciencia y las cosas no son como yo pienso o como yo quiero." [...] (Lupe)

Bergson, ${ }^{15}$ entre sus proposiciones relacionadas con el movimiento menciona que hay una realidad exterior $y$, no obstante, dada inmediatamente a nuestro espíritu, considerando que esta realidad es movilidad. No existen cosas hechas, sino sólo cosas que se hacen, no estados que se mantienen, sino sólo estados que cambian.

El reposo no es sino aparente, o mejor, relativo. La conciencia que tenemos de nuestra propia persona, en su continuo fluir, nos introduce en el interior de una realidad sobre el modelo del cual debemos representarnos las otras. Toda realidad es tendencia, si se conviene en llamar tendencia a un cambio de dirección en estado naciente. ${ }^{15}$
Bergson también hace referencia al espíritu que toma de vez en cuando vistas cuasi instantáneas sobre la movilidad indivisa de lo real; obtiene así sensaciones e ideas. De este modo, substituye lo continuo con lo discontinuo, la movilidad con la estabilidad, la tendencia en vía de cambio con los puntos fijos que señalan una dirección del cambio y de la tendencia a escapar lo que es su esencia misma. ${ }^{15}$

En la evolución de la vida se verifica, en efecto, un movimiento ascendente denominado impulso creador que produce formas cada vez más complejas y perfectas. ${ }^{16}$

\section{CONCLUSIONES}

En este estudio se observa que el espacio y movimiento de las actividades de cuidado se centran en realizar las actividades de la vida diaria que la persona con discapacidad no puede realizar de manera independiente; sin embargo, las formas de realizar estas actividades van transformándose de acuerdo con el tiempo y espacio que implica sucesión, continuidad y duración de éstas.

El movimiento que experimentan los cuidadores de personas con discapacidad motora puede ser ascendente, en el que se aprecian las características de un cuidado verdadero como es la paciencia y amor. Este movimiento ascendente permitirá al cuidador desarrollar su creatividad y establecer una relación transformadora con la persona con discapacidad motora.

\section{REFERENCIAS}

1. Organización Mundial para la Salud, Banco Mundial. Informe Mundial sobre la Discapacidad. Malta: Organización Mundial para la Salud; 2011.

2. Banco Mundial. Discapacidad y desarrollo inclusivo en América Latina y el Caribe 2009 [Internet]. [Acceso 14 julio 2012]. Disponible en: http:// web.worldbank.org/WBSITE/EXTERNAL/TOPICS/EXTSOCIALPROTECTION/EXTDISABILITY/0, contentMDK:20286156 pageP K:210058 piPK:210062 theSitePK:282699,00.html

3. Instituto Nacional de Estadística y Geografía. Principales resultados del Censo de Población y Vivienda 2010. México: INEGI; 2010.

4. Gutiérrez JP, Rivera-Dommarco J, Shamah-Levy T, Villalpando-Hernández S, Franco A, Cuevas-Nasu L, Romero-Martínez M, Hernández-Ávila M. Encuesta Nacional de Salud y Nutrición 2012. Resultados Nacionales. Cuernavaca, México: Instituto Nacional de Salud Pública (MX), 2012.

5. Montalvo-Prieto A, Florez-Torres IE, Vega DS. Cuidando a cuidadores familiares de niños en situación de discapacidad. Aquichán. 2008; 8 (2): 197-211.

6. Neves ET, Cabral IE. Cuidar de crianças com necessidades especiais de saúde: desafíos para as famílias e enfermagem pediática. Rev Eletr Enf [Internet]. 2009 [acceso 15 diciembre 2012]; 11(3):527-538g. Disponible en: http://www.fen.ufg.br./revista/v11/n3/v11n3ao9.htm

7. Rogero-García, J. El tiempo de cuidado familiar a mayores dependientes en España: entre la donación y la expropiación del tiempo. EKAINA 2011, Junio, 29-38. Doi:10.5569/1134-7147.49.03.

8. Flores GE, Rivas RE, Seguel PF. Nivel de sobrecarga en el desempeño del rol cuidador familiar de adulto mayor con dependencia severa. Ciencia y Enfermería. 2012; XVIII (1): 29-41.

9. Espinoza MK, Jofré AV. Sobrecarga, apoyo social y autocuidado en cuidadores informales. Ciencia y Enfermería. 2012; XVIII (2): 23-30.

10. Uesugui HM, Fagundes DS, Pinho DLM. Perfil e grau de dependência de idoso e sobrecarga de sus cuidadores. Acta Paul Enferm. 2011; 24 (5): 689-694. 
11. Gonçalves LHT, Alvarez AM, Sena ELS, Santana LWS, Vicente FR. Perfil da família cuidadora de idoso doente/fragilizado do contexto sociocultural de Florianópolis,SC. Texto Contexto Enferm. 2006; 15 (4): 570-577.

12. Schnaider TB, Silva JV, Pereira MA. Cuidador familiar de paciente com Afecção Neurológica. Saúde Soc. São Paulo. 2009; 18 (2): 282-292.

13. Bocchi, SCM, Silva L, Juliani CMCM, Spiri WC Familiares visitantes e acompanhantes de adultos e idosos hospitalizados: Análise da experiência na perspectiva do processo de trabalho em enfermagem. Rev. Latino-Am. Enfermagem [Internet]. 2007 [acceso 12 enero 2013]; 15 (2). Disponible en: http://www.eerp.usp.br/rlae
14. Correa IA. Conciencia, duración y tiempo como ejes epistemológicos En: Ciclo de Conferencias por el 150 Aniversario del Natalicio de Henri Bergson. 2009.

15. Bergson H. Introducción a la metafísica. México: Universidad Nacional Autónoma de México; 1960.

16. Santander J. El tiempo interrogado por los filósofos. Puebla: Benemérita Universidad Autónoma de Puebla; 1999.

17. Waldow VR. Nuevas ideas sobre el cuidado. Aquichán 2008; 6 (4): 85-96.

18. Bergson H. Ensayos sobre los datos inmediatos de la conciencia. Salamanca: Ediciones Sígueme; 1999. 